\title{
The Galactic interstellar medium: foregrounds and star formation
}

\author{
Marc-Antoine Miville-Deschênes ${ }^{1,2}$ \\ ${ }^{1}$ Laboratoire AIM, CEA / CNRS / Université Paris-Saclay, 91191, Gif-sur-Yvette, France \\ ${ }^{2}$ Institut d'Astrophysique Spatiale, CNRS / Université Paris-Sud, 91405, Orsay, France \\ email: mamd@cea.fr
}

\begin{abstract}
This review presents briefly two aspects of Galactic interstellar medium science that seem relevant for studying EoR. First, we give some statistical properties of the Galactic foreground emission in the diffuse regions of the sky. The properties of the emission observed in projection on the plane of the sky are then related to how matter is organised along the line of sight. The diffuse atomic gas is multi-phase, with dense filamentary structures occupying only about $1 \%$ of the volume but contributing to about $50 \%$ of the emission. The second part of the review presents aspect of structure formation in the Galactic interstellar medium that could be relevant for the subgrid physics used to model the formation of the first stars.
\end{abstract}

Keywords. ISM: kinematics and dynamics, magnetic fields, dust, reflection nebulae, structure

\section{Introduction}

The interstellar medium (ISM) is far from a homogenous atmosphere for our Galaxy. It is a turbulent, multiphase environment, with dramatic variations in temperature, density, magnetization, ionization, velocity, composition, and abundance. How matter evolves in a galaxy like the Milky Way, from diffuse and hot gas to dense and gravitationally stable structures producing stars, is not clear. In particular how energy, mass and momentum is transported from Galactic scales, through the different gas phases of the ISM and down to stellar scales, is still to be understood. This involves understanding the details of energy injection mechanisms at large scales, the interstellar mass and turbulence cascade, phase and chemical transitions, various instabilities, as well as the relative importance of gravity, stellar feedback, and cosmic-ray feedback at all scales. Because of the complex and non-linear physics involved in the evolution of matter in a galaxy like the Milky Way, it can be described as a self-organizing system where order arises from chaotic and random interactions. This field is currently the matter of intense research to investigate how exactly the gas flows from extragalactic scales down to individual stars. It plays a key role in understanding galaxy evolution in the Universe in general but also the origin of solar systems like our own.

In this short review, we address two topics relevant for the EoR field of research. First we present some informations about the statistical properties of the interstellar diffuse emission at high Galactic latitudes. This is relevant in the context of component separation and foreground cleaning. The second part of the paper presents elements of the star formation process in the Milky Way that might be helpful in the context of simulating the formation of the first stars. 


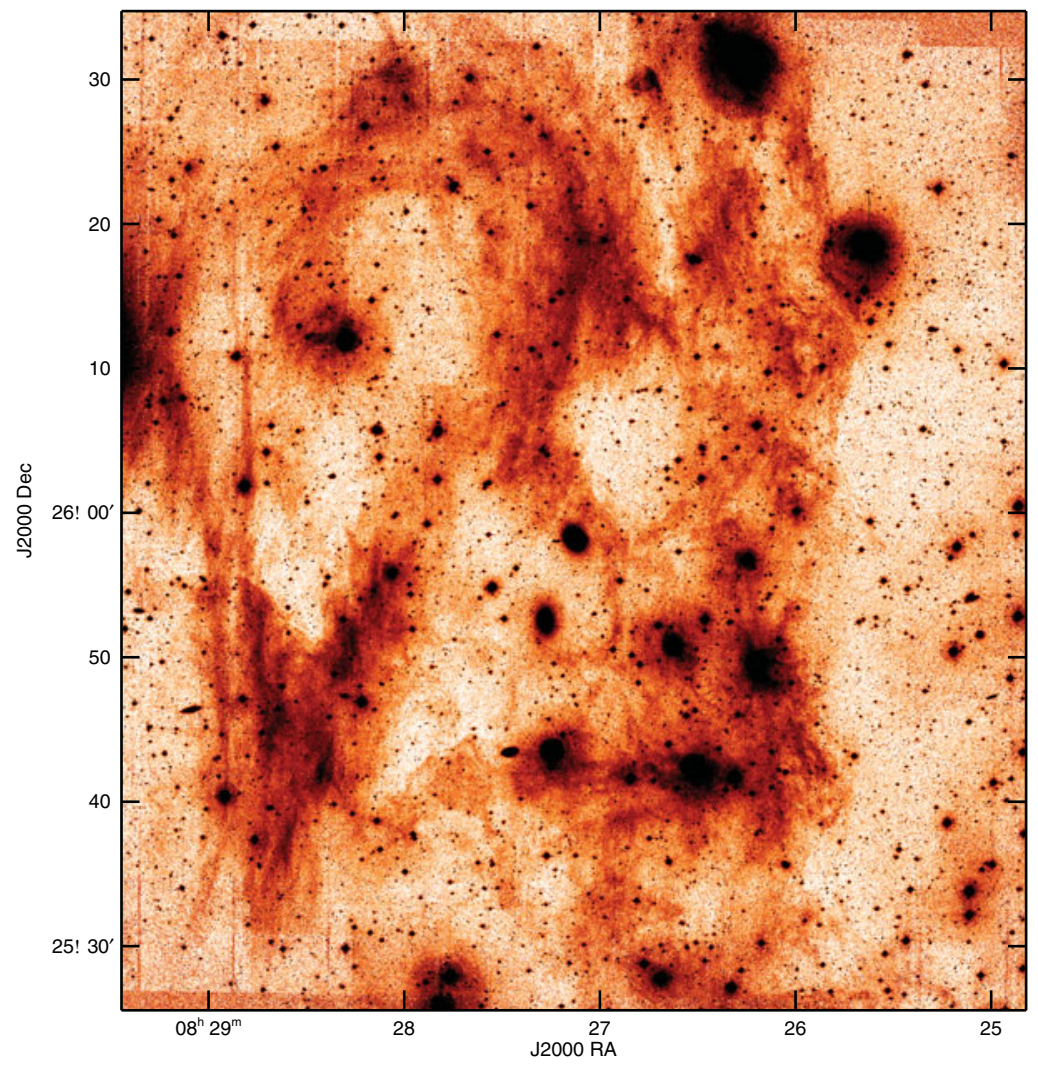

Figure 1. Diffuse optical light of a high Galactic latitude cirrus cloud (Miville-Deschênes et al. 2016). Observations obtained with MegaCam (g-band) on the CFHT, as part of the MATLAS program benefiting from a method that restore large scale diffuse emission (Duc et al. 2015).

\section{Statistical properties of the Galactic foreground emission}

\subsection{Power spectrum of interstellar structure}

The power spectrum of interstellar structure has been the subject of several studies since the pioneer work of Crovisier \& Dickey (1983) (see reviews by Elmegreen \& Scalo 2004 and Hennebelle \& Falgarone 2012). The ISM density structure is well characterized by a power law power spectrum with an exponent between -2.5 and -3.0 . Even though the slope of the power spectrum is a relatively poor information as it capturs a small fraction of the statistical information of the system, it can still be used to rule out some models.

One interesting way to trace the ISM structure is by looking at dust scattering in the visible and the UV, a method revisited recently by Boissier et al. (2015) and MivilleDeschênes et al. (2016) (see Fig. 1). In fact diffuse Galactic light, as it is called, has been observed in the optical since the 1930s. When observed with deep imaging surveys, it can be used as a tracer of the interstellar turbulent cascade, down to scales of about 1 arcsec. Miville-Deschênes et al. (2016) presented a power spectrum analysis of the dust column density of a diffuse cirrus at high Galactic latitude as derived from the combination of a MegaCam g-band image, obtained as part of the MATLAS large program at the CFHT, with Planck radiance and WISE $12 \mu \mathrm{m}$ data (see Fig. 2, left panel).

The combination of these three datasets have allowed them to compute the density power spectrum of the ISM structure over scales of more than three orders of magnitude. The density field is well described by a single power law over scales ranging from 0.01 to 

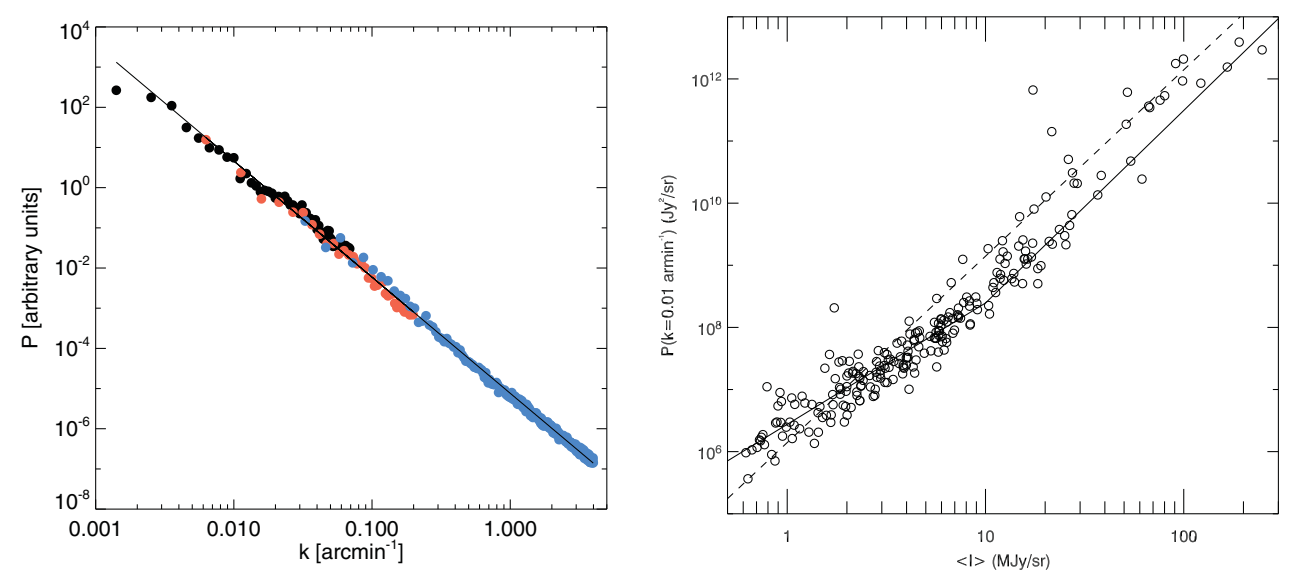

Figure 2. Left: Power spectrum of the diffuse region showed in Fig. 1. Blue dots are for MegaCam g-band, red dots for WISE and black dots for Planck radiance. See Miville-Deschênes et al. (2016) for details. Right: Normalisation of the power spectrum of dust $100 \mu \mathrm{m}$ emission for $12.5^{\circ} \times 12.5^{\circ}$ patches sampling the whole sky. From Miville-Deschênes et al. (2007).

$50 \mathrm{pc}$, with an exponent, $\gamma=-2.9 \pm 0.1$. This is typical of what is seen at high Galactic latitude. It is significantly different than what is predicted for sub-sonic or trans-sonic turbulence $(-3.7)$. On the other hand it is compatible with what is expected for thermally bi-stable and turbulent atomic gas (Saury et al. 2014). Moreover, no steepening of the power spectrum at small scales was found, indicating that the typical scale at which turbulent energy is dissipated in this medium is smaller than $0.01 \mathrm{pc}$. The ambipolar diffusion scenario that is sometimes proposed as a dissipative agent, is consistent with these observations only if the density of the cloud observed is higher than the typical values assumed for the cold neutral medium gas.

\subsection{Non-stationarity of the Galactic foreground emission}

Unlike the cosmic microwave background that is very well approximated by a Gaussian random field with a given power spectrum, the interstellar density field is not well described only by its second order moment distribution. The interstellar medium is far from a stationary Gaussian field; it has strong moments of order higher than two. One information that can be used to better describe the interstellar intensity fluctuations is encoded in the spatial variation of the normalisation of the power spectrum. Figure 2-right shows the power of the $100 \mu \mathrm{m}$ emission at a given scale (100 arcminutes), for different regions of the sky, as a function of their average $100 \mu \mathrm{m}$ brightness. This figure shows that the brightness fluctuations at a given scale are stronger in brighter regions of the sky. This comes as no surprise but, as shown by Miville-Deschênes et al. (2007), this encapsulates most of the information about the non-Gaussian and non-stationary behaviors of the Galactic emission.

The other obvious aspect of interstellar emission is the fact that it is not isotropic. Fluctuations have prefered orientations. In other words, the interstellar medium is somewhat filamentary. The exact nature of interstellar filaments is still not clear but it seems that 1) they are more present at small scales, 2) they are often related to the orientation of the magnetic field (see Fig. 3) and 3) they are present both in the non self-gravitating atomic gas as well as in gravitationnally bound molecular clouds.

To conclude, the interstellar structure is multi-scale, non-stationary and anisotropic. The power spectrum and the dependence of its normalisation with average brightness 


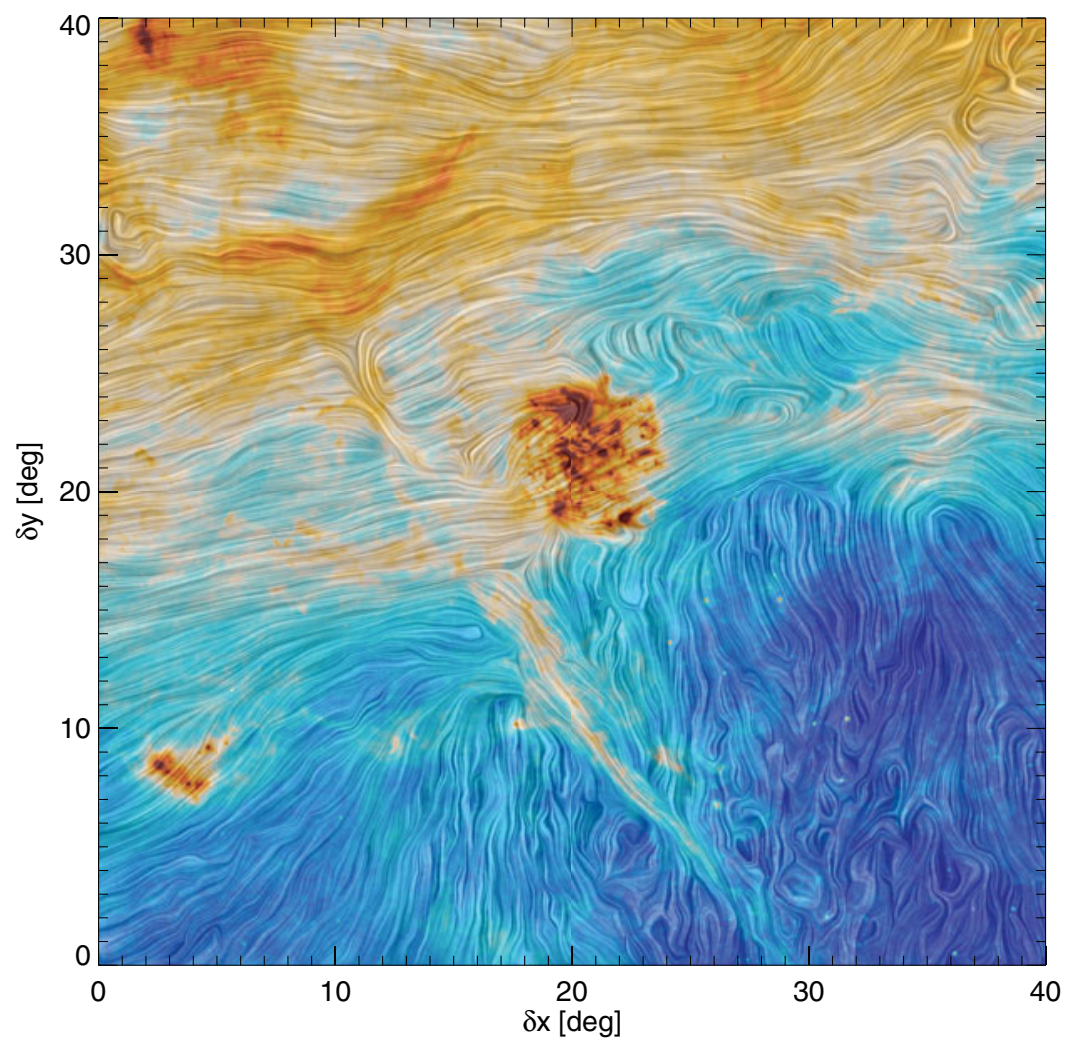

Figure 3. Dust emission and magnetic field orientation of a $40^{\circ} \times 40^{\circ}$ field centered on the Large Magellanic Cloud. The colour scale shows the dust radiance (Planck Collaboration 2014). The texture represents the magnetic field orientation calculated using the Line Integral Convolution algorithm applied to the Planck $353 \mathrm{GHz}$ polarization angle.

provide an interesting description of the statistical properties of the interstellar emission. On the other hand it is clearly incomplete as it does not carry any information about the filamentary structure of the ISM. This part of the statistical properties of the ISM has escaped characterization for now.

\subsection{The multi-phase nature of the diffuse ISM}

The Galactic interstellar medium at high Galactic latitude, where cosmological observations are performed, is dominated by the atomic phase. Actually, $80 \%$ of the sky seen from our vantage point has $A_{v}<1$ (Planck Collaboration 2014) which is the extinction at which molecular gas starts to be seen. Therefore, on the vast majority of lines of sight the gas is mostly in the neutral atomic phase (HI). The ubiquity of Galactic HI is revealed by its emission at $21 \mathrm{~cm}$ which is characterized by narrow features $\left(1-2 \mathrm{~km} \mathrm{~s}^{-1}\right)$ over wider smooth features $\left(5-10 \mathrm{~km} \mathrm{~s}^{-1}\right)$ which shows that HI is present in two different thermal states : a cold $(\sim 80 \mathrm{~K}-\mathrm{CNM})$ and a warm $(\sim 6000 \mathrm{~K}-\mathrm{WNM})$ phase (Fig. 4 left). The thermal bi-stability of the HI is the result of the specific dependence of the cooling and heating processes on gas density (Wolfire et al. 1995).

As both phases are roughly in pressure equilibrium, the large difference in temperature implies a density ratio of a factor about 100. At high Galactic latitudes, in low $A_{v}$ regions, the column density in each phase is about 50-50. If the column density is similar, and 

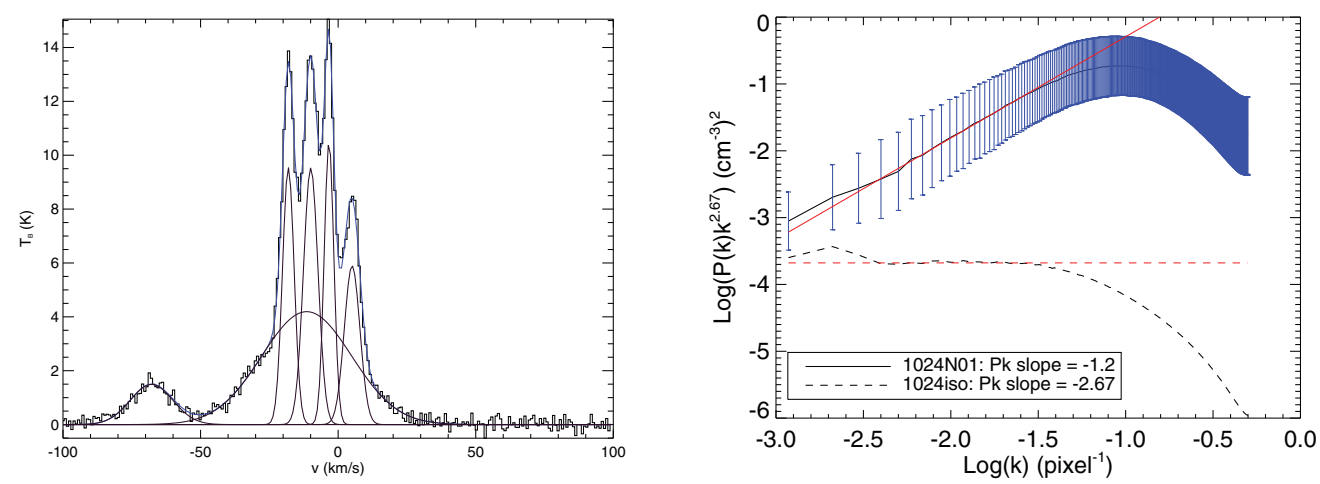

Figure 4. Left: Typical $21 \mathrm{~cm}$ spectrum of the Polaris Flare obtained with the Green Bank Telescope (Martin et al. 2015). The black histogram is the data, the black smooth lines represent the result of a Gaussian decomposition of the spectra, and the blue line is the sum of all Gaussians. Right: Power spectrum of the density for two numerical simulations: thermally bi-stable case (solid line) and isothermal case (dashed line). The spectra are multiplied by $k^{8 / 3}$ which is the prediction for isothermal, trans-sonic turbulence. The thermally bi-stable case has a significantly shallower slope, even though it is trans-sonic, caused by the formation of dense structures through the thermal instability. Taken from Saury et al. (2014).

the densities are a factor of 100 different, it implies that the volume filling factor of the CNM is about 1\%. This is also seen in numerical simulations (Saury et al. 2014).

Observations are indicating that the CNM is more structured at small scales with a newtork of very thin and elongated filament (Clark et al. 2014). Numerical simulations of thermally bi-stable HI, where the two phases can be separated without ambiguity, show that the power spectrum of the CNM density is significantly shallower that for isothermal trans-sonic turbulence (Fig. 4 right). In the specific case of HI, the large density contrast of cold structures is not the result of high Mach number turbulence like it might be the case in molecular clouds, but it is due to the interplay between sonic turbulence in the WNM and the thermal instability.

The observed statistical properties of the interstellar emission mentioned in the previous section are likely to be related to the specificity of the HI thermal and dynamical properties. The shallow power spectrum, the non-stationarity and the anisotropy of the Galactic emission are in part due to the fact that we are observing high-contrast and filamentary structures that fill only $1 \%$ of the volume, in projection on the sky.

\section{Elements of star formation in the Milky Way}

\subsection{Structure formation in the diffuse atomic phase}

As seen in the previous section, the formation of dense structures in the interstellar medium is a complex process. It is not solely driven by gravitationnal compression. In fact, the formation of dense (a few $\left.10^{2}-10^{3} \mathrm{~cm}^{-3}\right)$ and cold $(30-100 \mathrm{~K}$ ) CNM structures starts in the non self-gravitating atomic phase through the interplay between turbulence in the WNM and the thermal instability. Many numerical studies have shown that the production of CNM is more efficient when the pressure of the WNM is increased, in colliding flows for instance (e.g., Hennebelle \& Audit 2007). Observationnaly, such conditions are more difficult to identify.

One recent example is the Herschel observations of the Draco nebula (Fig. 5), a high Galactic latitude interstellar cloud that shows unusually strong CO emission and 


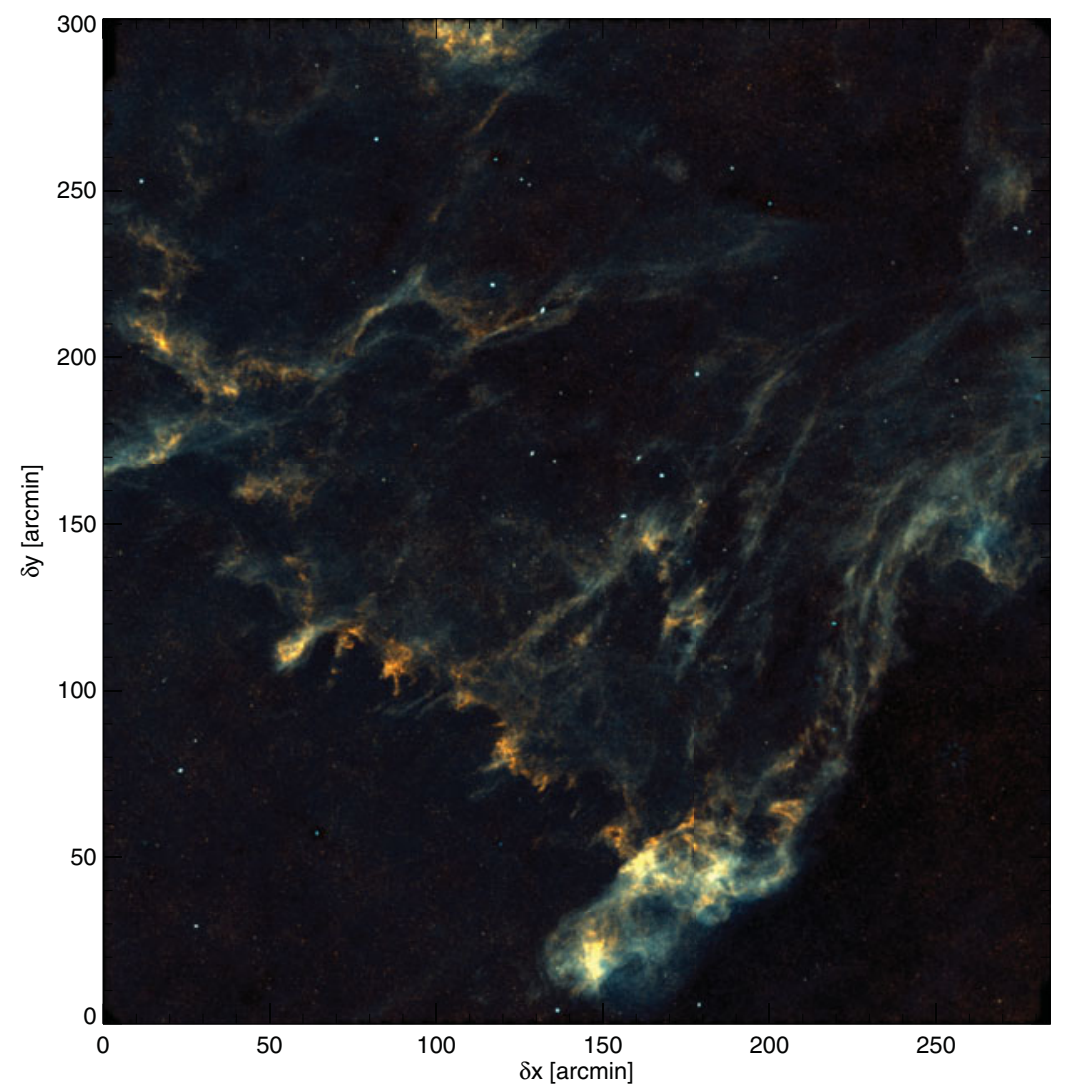

Figure 5. The Draco Nebula as observed with Herschel-SPIRE (250 $\mu \mathrm{m}$ - red) and WISE $(12 \mu \mathrm{m}-$ blue). The green channel of this RGB image is the average of the two others. From Miville-Deschênes et al. (2017b).

remarkably high-contrast small-scale structures for such a diffuse cloud (Miville-Deschênes et al. 2017b). The $21 \mathrm{~cm}$ emission of the Draco nebula reveals that it is likely to have been formed by the collision of a cloud entering the disk of the Milky Way. Such physical conditions are ideal to study the formation of cold and dense gas in colliding flows of diffuse and warm gas.

The high-resolution Herschel-SPIRE map reveals the fragmented structure of the interface between the infalling cloud and the Galactic layer. This front is reminiscent of a Rayleigh-Taylor instability structure. The statistical properties of the small-scale structures (typical size of $0.1-0.2 \mathrm{pc}$ ) are found to be typical of that seen in molecular clouds. The density of the gas has a log-normal distribution with an average value of $10^{3} \mathrm{~cm}^{-3}$, but contrary to molecular clouds, only $15 \%$ of the mass of the cloud is in gravitationally bound structures. Here, the formation of dense structures is the result of the interplay between magnetohydrodynamical turbulence and thermal instability as self-gravity is not dominating the dynamics. The increase of pressure of the warm gas, induced by the collision, is strong enough to trigger the formation of cold and dense structures, ready to form stars, showing that this process can happen even though self-gravity is not a dominant part of the total energy. 

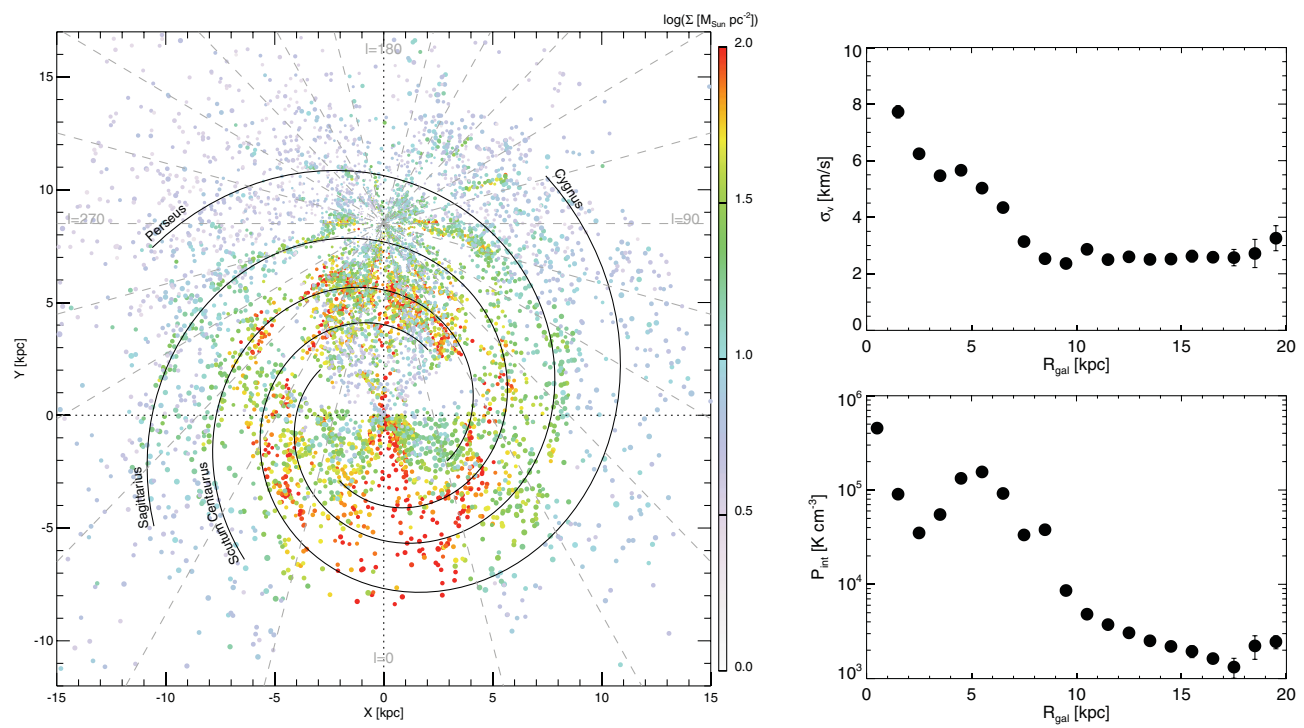

Figure 6. Left: Representation of the molecular cloud surface density on a Galactic top view diagram (Galactic center at $[0,0]$; Sun at $[0,8.5]$ ). Right: Average velocity dispersion (top) and average pressure (bottom) of molecular clouds as a function of Galacto-centric radius. From Miville-Deschênes et al. (2017a).

\subsection{Properties of molecular clouds}

Molecular clouds are the sites of all present-day star formation in the Galaxy. The mass in their densest cores $\left(>10^{6} \mathrm{~cm}^{-3}\right)$ can be high enough that they become gravitationally unstable with respect to thermal and magnetic support. Understanding the properties of molecular clouds, their variations across the Galaxy and their link with the star formation activity is still a very active field of research.

A recent analysis of low resolution archived ${ }^{12} \mathrm{CO}$ data of the Milky Way (Dame et al. 2001) has allowed the production of the most complete catalog of molecular clouds to date (Miville-Deschênes et al. 2017a). This study presents a catalog of 8107 molecular clouds that covers the entire Galactic plane (see Fig. 6 left). It includes $98 \%$ of the ${ }^{12} \mathrm{CO}$ emission observed within $\pm 5^{\circ}$ of Galactic latitude, compared to $20-25 \%$ for previous studies. The catalog was produced using a hierarchical cluster identification method applied to the result of a Gaussian decomposition of the $\mathrm{CO}$ data. The total $\mathrm{H}_{2}$ mass in the catalog is $1.2 \times 10^{9} \mathrm{M}_{\odot}$, in agreement with previous estimates.

Even though such studies are limited by the fact that we observed the multi-scale ISM from within our Galaxy (i.e, clouds that are closer are broken up in smaller pieces) the significant step forward in completeness makes possible to study variations of the properties of molecular clouds with Galacto-centric radius, $R_{\text {gal }}$. In contrast with the general idea, there is a rather large range of values of the molecular clouds surface density, $\Sigma$, from 2 to $300 \mathrm{M}_{\odot} \mathrm{pc}^{-2}$, and a systematic decrease with increasing $R_{\text {gal }}$ (see Fig. 2 left panel). The cloud velocity dispersion and the normalization $\sigma_{0}=\sigma_{v} / R^{1 / 2}$ both decrease systematically with $R_{\text {gal }}$ (see Fig. 6 top-right).

When studied over the whole Galactic disk, there is a large dispersion in the line widthsize relation and a significantly better correlation between $\sigma_{v}$ and $\Sigma R$. The normalization of this correlation is constant to better than a factor of two for $R_{\text {gal }}<20 \mathrm{kpc}$. On the other hand, strong radial variation of the turbulent energy injection rate and of the internal pressure are found (see Fig. 6 bottom-right). The increase in pressure in the 
inner part of the Galaxy coincides with the region where star formation is observed. This pressure increase could be due to a combination of kinematic energy injected by the star formation activity itself (positive feedback) and of an increase of the Galactic gravitational potential energy due to the higher stellar mass. In the outer Galaxy the energy injection rate may be maintained by accretion through the disk and/or onto the clouds.

\section{Conclusion}

The star formation process in galaxies is the results of a combination of complex physical processes, some happening at the atomic scale (heating, cooling, dissipation of kinematic and magnetic energy) and some affecting the medium over a broad range of scales (MHD turbulence, gravity, radiation). The interstellar medium structure reveals this complexy which can be a challenge for extra-galactic and cosmological studies. In this review we have shown that

(a) Galactic emission is well described by a power law power spectrum, down to arcsecond scales, but that is not enough to characterize its statistical properties fully. Galactic structure is also non-stationary and anisotropic.

(b) The high Galactic latitude sky is composed of a small number of dense, cold and anisotropic structures that fill only $1 \%$ of the volume. They are embedded in a warm medium, partly neutral and partly ionized. The mass fraction of the cold and warm phases in the diffuse regions of the sky is about $50-50 \%$.

The ISM of the Milky Way brings detailed informations about the dense structure formation process which could be useful to better model the formation of the first stars.

(a) Structure formation in the ISM starts in non-self gravitating but over-pressured warm regions, by a combination of turbulence and thermal instability.

(b) Star formation then occurs in self-gravitating structures formed out of the previously structured ISM. The gravitational collapse is hierarchical, where small scale structures collapse first.

\section{References}

Boissier, S., Boselli, A., Voyer, E., et al. 2015, A\& $A$, 579, A29

Clark, S. E., Peek, J. E. G., \& Putman, M. E. 2014, ApJ, 789, 82

Crovisier, J. \& Dickey, J. M. 1983, A\&GA, 122, 282

Dame, T. M., Hartmann, D., \& Thaddeus, P. 2001, ApJ, 547, 792

Duc, P.-A., Cuillandre, J.-C., Karabal, E., et al. 2015, MNRAS, 446, 120

Elmegreen, B. G. \& Scalo, J. 2004, ARA\&A, 42, 211

Hennebelle, P. \& Audit, E. 2007, A\&A, 465, 431

Hennebelle, P. \& Falgarone, E. 2012, A\&ARv, 20, 55

Martin, P. G., Blagrave, K. P. M., Lockman, F. J., et al. 2015, ApJ, 809, 153

Miville-Deschênes, M.-A., Duc, P.-A., Marleau, F., et al. 2016, A\&\&A, 593, A4

Miville-Deschênes, M.-A., Lagache, G., Boulanger, F., \& Puget, J.-L. 2007, A\&A, 469, 595

Miville-Deschênes, M.-A., Murray, N., \& Lee, E. J. 2017a, ApJ, 834, 57

Miville-Deschênes, M.-A., Salomé, Q., Martin, P. G., et al. 2017b, A\&\&A, 599, A109

Planck Collaboration, Abergel, A., Ade, P. A. R., et al. 2014, A\& A, 571, A11

Saury, E., Miville-Deschênes, M.-A., Hennebelle, P., Audit, E., \& Schmidt, W. 2014, A\&A A, 567, A16

Wolfire, M. G., Hollenbach, D., McKee, C. F., Tielens, A. G. G. M., \& Bakes, E. L. O. 1995, ApJ, 443, 152 